International Journal of Pure and Applied Mathematics

Volume 84 No. 5 2013, 463-476

ISSN: 1311-8080 (printed version); ISSN: 1314-3395 (on-line version)

url: http://www.ijpam.eu

doi: http://dx.doi.org/10.12732/ijpam.v84i5.2

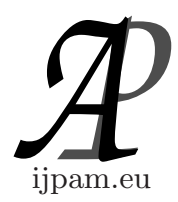

\title{
A RADIUS OF ABSOLUTE CONVERGENCE FOR MULTIVARIATE POWER SERIES
}

\author{
U.Dj. Bekbaev \\ Turin Polytechnic University in Tashkent \\ INSPEM, Universiti Putra Malaysia \\ MALAYSIA
}

\begin{abstract}
In this paper a new (symmetric) product for matrices, entries of which are located by pair of multi-indices, and norms of such matrices are introduced. They are used to represent multivariate power series as series in one (vector) variable and to introduce a radius of absolute convergence. For the radius of absolute convergence a formula, similar to ordinary Cauchy-Hadamard formula in one variable case, is given. Some open problems related to the radius of absolute convergence are set.
\end{abstract}

AMS Subject Classification: 32A05, 15A60

Key Words: multi-index, multivariate power series

\section{Introduction}

In theory of multivariate power series, see [1], for power series

$$
\sum_{\alpha} a_{\alpha} z^{\alpha}
$$

, where $\alpha=\left(\alpha_{1}, \alpha_{2}, \ldots, \alpha_{n}\right)$ runs all $n$ - tuples with nonnegative integer components, $a_{\alpha}$ are complex numbers, $z=\left(z_{1}, z_{2}, \ldots, z_{n}\right), z^{\alpha}=z_{1}^{\alpha_{1}} z_{2}^{\alpha_{2}} \ldots z_{n}^{\alpha_{n}}$, the formula

$$
\varlimsup_{|\alpha| \rightarrow \infty}\left(\left|a_{\alpha} r^{\alpha}\right|\right)^{\frac{1}{|\alpha|}}=1
$$

is called the space analog of the Cauchy-Hadamard formula, where $r=\left(r_{1}, r_{2}, \ldots\right.$,

Received: September 5, 2011

(c) 2013 Academic Publications, Ltd. url: www.acadpubl.eu 
$\left.r_{n}\right), r_{i}$ are so called adjoint radii of convergence for power series (1).

In this paper we are going to offer a radius of absolute convergence for multivariate power series. More exactly, for any $\rho \geq 1$ we consider the corresponding $\rho$-norm and offer for each multivariate power series (1) nonnegative numbers (or $+\infty) R_{\varrho}, R_{\varrho, c}=R_{\varrho} n^{\frac{1}{\varrho}}$, where $\frac{1}{\rho}+\frac{1}{\varrho}=1$, for which the following is true. Power series (1) is absolute convergent at $z$ whenever $\|z\|_{\varrho}<R_{\varrho}$ and for any $R>R_{\varrho, c}$ there exists such $\|z\|_{\varrho}=R$ at which the power series is absolute divergent. At the end of the paper we consider some examples of multivariate power series to demonstrate effectiveness the offered radius of absolute convergence and set some open problems.

To prove the above mentioned result we represent multivariate power series as a power series in one (vector) variable by introducing a new product $\odot$ for matrices, which can be called the symmetric product, define $\rho$-norm for matrices and investigate its properties with respect to the ordinary and symmetric products of matrices. Some main properties of the symmetric product also are presented here in order to draw attention of specialists to this useful operation. For more information and applications of the symmetric product one can see [2] and [3]. The main result on radius of absolute convergence for multivariate power series is announced in [4].

This paper is organized as follows. In Section 2, we introduce the symmetric product $\odot$ of matrices, study its some main properties which will be used in the next section. In Section 3, we introduce a radius of absolute convergence for multivariate power series by a formula similar to the Cauchy-Hadamard formula for one variable case and prove the main result of this paper (Theorem $2)$. In this section one can find some open problems as well.

\section{The Symmetric Product of Matrices}

Here are some definitions and results related to the new product introduced in $[2]$.

For a positive integer $n$ let $I_{n}$ stand for all row $n$-tuples with nonnegative integer entries with the following linear order: $\beta=\left(\beta_{1}, \beta_{2}, \ldots, \beta_{n}\right)<\alpha=$ $\left(\alpha_{1}, \alpha_{2}, \ldots, \alpha_{n}\right)$ if and only if $|\beta|<|\alpha|$ or $|\beta|=|\alpha|$ and $\beta_{1}>\alpha_{1}$ or $|\beta|=|\alpha|$, $\beta_{1}=\alpha_{1}$ and $\beta_{2}>\alpha_{2}$ etcetera, where $|\alpha|$ stands for $\alpha_{1}+\alpha_{2}+\ldots+\alpha_{n}$.

It is clear that for $\alpha, \beta, \gamma \in I_{n}$ one has $\alpha<\beta$ if and only if $\alpha+\gamma<\beta+\gamma$, where the sum means the component-wise sum. We write $\beta \ll \alpha$ if $\beta_{i} \leq \alpha_{i}$ for all $i=1,2, \ldots, n,\left(\begin{array}{c}\alpha \\ \beta\end{array}\right)$ stands for $\frac{\alpha !}{\beta !(\alpha-\beta) !}, \alpha !=\alpha_{1} ! \alpha_{2} ! \ldots \alpha_{n} !$ 
In future $n$ and $n^{\prime}$ are assumed to be any fixed positive integers. Let $F$ stand for the field of real or complex numbers.

For any nonnegative integer numbers $p, p^{\prime}$ let $M_{n^{\prime}, n}\left(p^{\prime}, p ; F\right)=M\left(p^{\prime}, p ; F\right)$ stand for all " $p^{\prime} \times p$ " size matrices $A=\left(A_{\alpha}^{\alpha^{\prime}}\right)_{|\alpha|=p,\left|\alpha^{\prime}\right|=p^{\prime}}$ ( $\alpha^{\prime}$ presents row, $\alpha$ presents column and $\left.\alpha \in I_{n}, \alpha^{\prime} \in I_{n^{\prime}}\right)$ with entries from $F$.

The ordinary size of such matrix is $\left(\begin{array}{c}p^{\prime}+n^{\prime}-1 \\ n^{\prime}-1\end{array}\right) \times\left(\begin{array}{c}p+n-1 \\ n-1\end{array}\right)$. Over such kind matrices in addition to the ordinary sum and product of matrices we consider the following "product" $\odot$ as well:

Definition 1. If $A \in M\left(p^{\prime}, p ; F\right)$ and $B \in M\left(q^{\prime}, q ; F\right)$ then $A \odot B=$ $C \in M\left(p^{\prime}+q^{\prime}, p+q ; F\right)$ such that for any $|\alpha|=p+q,\left|\alpha^{\prime}\right|=p^{\prime}+q^{\prime}$, where $\alpha \in I_{n}, \alpha^{\prime} \in I_{n^{\prime}}$,

$$
C_{\alpha}^{\alpha^{\prime}}=\sum_{\beta, \beta^{\prime}}\left(\begin{array}{c}
\alpha \\
\beta
\end{array}\right) A_{\beta}^{\beta^{\prime}} B_{\alpha-\beta}^{\alpha^{\prime}-\beta^{\prime}}
$$

, where the sum is taken over all $\beta \in I_{n}, \beta^{\prime} \in I_{n^{\prime}}$, for which $|\beta|=p,\left|\beta^{\prime}\right|=p^{\prime}$, $\beta \ll \alpha$ and $\beta^{\prime} \ll \alpha^{\prime}$.

Example 1. If $n=n^{\prime}=2, p=p^{\prime}=q=q^{\prime}=1$ in ordinary notations for matrices $A=\left(\begin{array}{ll}a_{11} & a_{12} \\ a_{21} & a_{22}\end{array}\right), B=\left(\begin{array}{ll}b_{11} & b_{12} \\ b_{21} & b_{22}\end{array}\right)$ the product $A \odot B$ can be given as

$$
\begin{aligned}
& A \odot B= \\
& \left(\begin{array}{ccc}
2 a_{11} b_{11} & a_{11} b_{12}+a_{12} b_{11} & 2 a_{12} b_{12} \\
2\left(a_{11} b_{21}+a_{21} b_{11}\right) & a_{11} b_{22}+a_{22} b_{11}+a_{12} b_{21}+a_{21} b_{12} & 2\left(a_{12} b_{22}+a_{22} b_{12}\right) \\
2 a_{21} b_{21} & a_{21} b_{22}+a_{22} b_{21} & 2 a_{22} b_{22}
\end{array}\right)
\end{aligned}
$$

It is hoped that the introduced product $\odot$ deserves the honor to be called the symmetric product. In [2] a matrix representation for polynomial maps is offered and by the use of the sum, ordinary product and symmetric product the matrix representation for composition of polynomial maps is given. The appearance of the symmetric product in composition of maps one can consider as an explanation of the fractal phenomenon. Indeed in symmetric powers of a given matrix one can see fractals in numbers.

Here we would like to present some important properties of the symmetric product, when proofs are not difficult they are omitted.

Let us agree that $h(H, v, V)$ stands for any element of $M(0,1 ; F)$ (respect. $M(0, p ; F), M(1,0 ; F), M(p, 0 ; F)$, where $p$ may be any nonnegative integer). 
For the sake of convenience it will be assumed that $A_{\alpha}^{\alpha^{\prime}}=0(\alpha !=\infty)$ whenever $\alpha \notin I_{n}$ or $\alpha^{\prime} \notin I_{n^{\prime}}$ (respect. $\alpha \notin I_{n}$ ).

Proposition 1. For the above defined product the following are true.

1. $A \odot B=B \odot A$.

2. $(A+B) \odot C=A \odot C+B \odot C$.

3. $(A \odot B) \odot C=A \odot(B \odot C)$

4. $(\lambda A) \odot B=\lambda(A \odot B)$ for any $\lambda \in F$

5. If $A$ and $B$ are square upper triangular matrices then $A \odot B$ is also an upper triangular matrix.

6. $A \odot B=0$ if and only if $A=0$ or $B=0$.

7. $(A \odot V) B=(A B) \odot V$

8. $A(B \odot H)=(A B) \odot H$

In future $A^{(m)}$ means the $m$-th power of matrix $A$ with respect to the symmetric product.

Proposition 2. If $h=\left(h_{1}, h_{2}, \ldots, h_{n}\right) \in M(0,1 ; F), v=\left(v_{1}, v_{2}, \ldots, v_{n}\right) \in$ $M(1,0 ; F)$, then

$$
\left(h^{(m)}\right)_{\alpha}^{0}=m ! h^{\alpha}, \quad\left(v^{(m)}\right)_{0}^{\alpha}=\left(\begin{array}{c}
m \\
\alpha
\end{array}\right) v^{\alpha},
$$

where $v^{\alpha}$ stands for $v_{1}^{\alpha_{1}} v_{2}^{\alpha_{2}} \ldots v_{n}^{\alpha_{n}}$.

Proposition 3. For any nonnegative integers $p, q, p^{\prime}, q^{\prime}$ and matrices $A \in M_{n^{\prime}, n}\left(p^{\prime}, p ; F\right), B \in M_{n^{\prime}, n}\left(q^{\prime}, q ; F\right), h=\left(h_{1}, h_{2}, \ldots, h_{n^{\prime}}\right) \in M_{n^{\prime}, n^{\prime}}(0,1 ; F)$, $v=\left(v_{1}, v_{2}, \ldots, v_{n}\right) \in M_{n, n}(1,0 ; F)$ the following equalities

$$
\begin{aligned}
\left(\frac{h^{\left(p^{\prime}\right)}}{p^{\prime} !} A\right) \bigodot\left(\frac{h^{\left(q^{\prime}\right)}}{q^{\prime} !} B\right) & =\frac{h^{\left(p^{\prime}+q^{\prime}\right)}}{\left(p^{\prime}+q^{\prime}\right) !}(A \bigodot B), \\
\left(A \frac{v^{(p)}}{p !}\right) \bigodot\left(B \frac{v^{(q)}}{q !}\right) & =(A \bigodot B) \frac{v^{(p+q)}}{(p+q) !}
\end{aligned}
$$

are true.

We consider the following $\rho$-norm of elements $A \in M\left(p^{\prime}, p ; F\right)$ :

\section{Definition 2.}

$$
\|A\|=\|A\|_{\rho}=\left(\sum_{\alpha, \alpha^{\prime}} \frac{\left|A_{\alpha}^{\alpha^{\prime}}\right|^{\rho}}{\alpha !\left(p ! p^{\prime} !\right)^{\rho-1}}\right)^{1 / \rho}
$$


In the case of $\rho=\infty$ the norm is defined as

$$
\|A\|=\|A\|_{\infty}=\frac{\max \left|A_{\alpha}^{\alpha^{\prime}}\right|}{p ! p^{\prime} !}
$$

Theorem 1. 1. If $A, B \in M\left(p^{\prime}, p ; F\right)$ and $\lambda \in F$ then:

a) $\|A\|=0$ if and only if $A=0$,

b) $\|\lambda A\|=|\lambda|\|A\|$,

c) $\|A+B\| \leq\|A\|+\|B\|$.

2. For any nonnegative integer numbers $p, p^{\prime}, q$ and $q^{\prime}$ there is such positive number $\lambda\left(p, p^{\prime}, q, q^{\prime}\right)$ that for any $A \in M\left(p^{\prime}, p ; F\right), B \in M\left(q^{\prime}, q ; F\right)$ the following inequality is valid:

$$
\lambda\left(p, p^{\prime}, q, q^{\prime}\right)\|A\|\|B\| \leq\|A \bigodot B\| \leq\|A\|\|B\| .
$$

Proof. Here is a proof of part 2. First let us show the inequality $\|A \odot B\| \leq$ $\|A\|\|B\|$.

Due to the Hölder inequality for $A \odot B=C$ one has

$$
\begin{gathered}
\left|C_{\alpha}^{\alpha^{\prime}}\right|=\left|\sum_{\beta \ll \alpha, \beta^{\prime} \ll \alpha^{\prime}}\left(\begin{array}{c}
\alpha \\
\beta
\end{array}\right) A_{\beta}^{\beta^{\prime}} B_{\alpha-\beta}^{\alpha^{\prime}-\beta^{\prime}}\right| \leq \\
\alpha ! \sum_{\beta \ll \alpha, \beta^{\prime} \ll \alpha^{\prime}} \frac{\left|A_{\beta}^{\beta^{\prime}} B_{\alpha-\beta}^{\alpha^{\prime}-\beta^{\prime}}\right|}{(\beta !(\alpha-\beta) !)^{1 / \rho}} \frac{1}{(\beta !(\alpha-\beta) !)^{1-1 / \rho}} \leq \\
\alpha !\left(\sum_{\beta \ll \alpha, \beta^{\prime} \ll \alpha^{\prime}} \frac{\left|A_{\beta}^{\beta^{\prime}} B_{\alpha-\beta}^{\alpha^{\prime}-\beta^{\prime}}\right| \rho}{\beta !(\alpha-\beta) !}\right)^{1 / \rho}\left(\sum_{\beta \ll \alpha, \beta^{\prime} \ll \alpha^{\prime}}\left(\frac{1}{(\beta !(\alpha-\beta) !)^{1-1 / \rho}}\right)^{\varrho}\right)^{1 / \varrho}= \\
\alpha !\left(\sum_{\beta \ll \alpha, \beta^{\prime} \ll \alpha^{\prime}} \frac{\left|A_{\beta}^{\beta^{\prime}}\right| \rho\left|B_{\alpha-\beta}^{\alpha^{\prime}-\beta^{\prime}}\right| \rho}{\beta !} \frac{1}{(\alpha-\beta) !}\right)^{1 / \rho}\left(\sum_{\beta \ll \alpha} \frac{1}{\beta !(\alpha-\beta) !} \sum_{\beta^{\prime} \ll \alpha^{\prime}} 1\right)^{1 / \varrho} \leq \\
\alpha !\left(\sum_{\beta \ll \alpha, \beta^{\prime} \ll \alpha^{\prime}} \frac{\left|A_{\beta}^{\beta^{\prime}}\right| \rho}{\beta !} \frac{\left|B_{\alpha-\beta}^{\alpha^{\prime}-\beta^{\prime}}\right| \rho}{(\alpha-\beta) !}\right)^{1 / \rho}\left(\left(\begin{array}{c}
p+q \\
p
\end{array}\right) \frac{1}{\alpha !}\left(\begin{array}{c}
p^{\prime}+q^{\prime} \\
p^{\prime}
\end{array}\right)\right)^{1 / \varrho}
\end{gathered}
$$

as far as $\sum_{\beta \ll \alpha} \frac{1}{\beta !(\alpha-\beta) !}=\left(\begin{array}{c}p+q \\ p\end{array}\right) \frac{1}{\alpha !}$ and $\sum_{\beta^{\prime} \ll \alpha^{\prime}} 1 \leq\left(\begin{array}{c}p^{\prime}+q^{\prime} \\ p^{\prime}\end{array}\right)$. Therefore

$$
\|C\|=\left(\sum_{\alpha, \alpha^{\prime}} \frac{\left|C_{\alpha}^{\alpha^{\prime}}\right|^{\rho}}{\alpha !\left((p+q) !\left(p^{\prime}+q^{\prime}\right) !\right)^{\rho-1}}\right)^{1 / \rho} \leq
$$




$$
\begin{gathered}
\left(\sum_{\alpha, \alpha^{\prime}} \frac{1}{\alpha !\left((p+q) !\left(p^{\prime}+q^{\prime}\right) !\right)^{\rho-1}}(\alpha !)^{\rho}\right. \\
\left.\sum_{\beta \ll \alpha, \beta^{\prime} \ll \alpha^{\prime}} \frac{\left|A_{\beta}^{\beta^{\prime}}\right|^{\rho}}{\beta !} \frac{B_{\alpha-\beta}^{\alpha^{\prime}-\beta^{\prime}} \mid \rho}{(\alpha-\beta) !}\left(\left(\begin{array}{c}
p+q \\
p
\end{array}\right) \frac{1}{\alpha !}\left(\begin{array}{c}
p^{\prime}+q^{\prime} \\
p^{\prime}
\end{array}\right)\right)^{\rho / \varrho}\right)^{1 / \rho}= \\
\left(\sum_{\beta \beta^{\prime}} \frac{\left|A_{\beta}^{\beta^{\prime}}\right|^{\rho}}{\beta !\left(p ! p^{\prime} !\right)^{\rho-1}}\right)^{1 / \rho}\left(\sum_{\gamma \gamma^{\prime}} \frac{\left|B_{\gamma}^{\gamma^{\prime}}\right|^{\rho}}{\gamma !\left(q ! q^{\prime} !\right)^{\rho-1}}\right)^{1 / \rho}=\|A\|\|B\|
\end{gathered}
$$

due to $\rho / \varrho=\rho-1$

To show the inequality $\lambda\left(p, p^{\prime}, q, q^{\prime}\right)\|A\|\|B\| \leq\|A \odot B\|$ let us consider

$$
X=\left\{(A, B): A \in M\left(p^{\prime}, p ; F\right),\|A\|_{\rho}=1, B \in M\left(q^{\prime}, q ; F\right),\|B\|_{\rho}=1\right\}
$$

, which is a compact set in the corresponding finite dimensional vector space, and the continuous map $(A, B) \mapsto A \odot B$. The image of $X$ with respect to this map is a compact set which doesn't contain zero vector due to Proposition 1. Let $\lambda\left(p, p^{\prime}, q, q^{\prime}\right)>0$ stand for the distance between zero vector and this image set with respect to the corresponding $\rho$ - norm. So $\lambda\left(p, p^{\prime}, q, q^{\prime}\right) \leq\|A \odot B\|_{\rho}$ for any $(A, B) \in X$ and due to Proposition 1 one has

$$
\lambda\left(p, p^{\prime}, q, q^{\prime}\right)\|A\|\|B\| \leq\|A \bigodot B\|
$$

for any $A \in M\left(p^{\prime}, p ; F\right), B \in M\left(q^{\prime}, q ; F\right)$

With respect to the ordinary product of matrices a result similar to

$$
\|A \bigodot B\| \leq\|A\|\|B\|
$$

is not valid. But one can have the following result.

Proposition 4. The following inequality

$$
\left\|A\left(q^{\prime}, q\right) B(q, p)\right\| \leq\left(q^{\prime} !\right)^{2 / \rho-1}(q !)^{2-1 / \rho}\|A\|_{\varrho}\|B\|_{\rho}
$$

is true.

Proof. Indeed due to the Hölder inequality one has

$$
\begin{gathered}
\left\|A\left(q^{\prime}, q\right) B(q, p)\right\|^{\rho}=\sum_{\alpha, \alpha^{\prime}} \frac{1}{\alpha !\left(p ! q^{\prime !}\right)^{\rho-1}}\left|\sum_{\beta} A_{\beta}^{\alpha^{\prime}} B_{\alpha}^{\beta}\right|^{\rho} \leq \\
\sum_{\alpha, \alpha^{\prime}} \frac{1}{\alpha !\left(p ! q^{\prime} !\right)^{\rho-1}}\left(\sum_{\beta}\left|A_{\beta}^{\alpha^{\prime}}\right|^{\varrho}\right)^{\rho / \varrho} \sum_{\gamma}\left|B_{\alpha}^{\gamma}\right|^{\rho}=
\end{gathered}
$$




$$
\left(\sum_{\alpha^{\prime}, \beta} \frac{\left|A_{\beta}^{\alpha^{\prime}}\right| \varrho}{\beta !\left(q^{\prime} ! q !\right)^{\varrho-1}} \beta !\right)^{\rho / \varrho}(q !)^{\rho}\left(q^{\prime} !\right)^{2-\rho} \sum_{\gamma, \alpha} \frac{\left|B_{\alpha}^{\gamma}\right|^{\rho}}{\alpha !(q ! p !)^{\rho-1}} \leq\|A\|_{\varrho}^{\rho}\|B\|_{\rho}^{\rho}(q !)^{2 \rho-1}\left(q^{\prime} !\right)^{2-\rho}
$$

as far as $\beta ! \leq q !$.

In particular case the following estimation is also true.

Proposition 5. For any nonnegative integer numbers $m, k, p^{\prime}$ and $v \in$ $M_{n, n}(1,0 ; F)$, $A \in M_{n^{\prime}, n}\left(p^{\prime}, m+k ; F\right)$ the following inequality

$$
\left\|A\left(\frac{v^{(m)}}{m !} \bigodot E_{k}\right)\right\|_{\rho} \leq\left(\begin{array}{c}
m+k \\
k
\end{array}\right)\|A\|_{\rho}\|v\|_{\varrho}^{m}
$$

is valid, where $E_{k}$ stands for " $k \times k$ " size unit matrix.

Proof. Indeed

$$
\begin{aligned}
\left\|A\left(p^{\prime}, m+k\right)\left(\frac{v^{(m)}}{m !} \bigodot E_{k}\right)\right\|_{\rho}^{\rho}=\sum_{\alpha, \alpha^{\prime}} \frac{1}{\alpha !\left(p^{\prime} ! k !\right)^{\rho-1}}\left|\left(A\left(\frac{v^{(m)}}{m !} \bigodot E_{k}\right)\right)_{\alpha}^{\alpha^{\prime}}\right|^{\rho}= \\
\sum_{\alpha, \alpha^{\prime}} \frac{1}{\alpha !\left(p^{\prime} ! k !\right)^{\rho-1}}\left|\sum_{\beta} A_{\beta}^{\alpha^{\prime}}\left(\frac{v^{(m)}}{m !} \bigodot E_{k}\right)_{\alpha}^{\beta}\right|^{\rho}= \\
\sum_{\alpha, \alpha^{\prime}} \frac{1}{\alpha !\left(p^{\prime} ! k !\right)^{\rho-1}}\left|\sum_{\beta \gg \alpha} \frac{A_{\beta}^{\alpha^{\prime}}}{(\beta-\alpha) !^{1 / \rho}} \frac{v^{\beta-\alpha}}{(\beta-\alpha) !^{1 / \varrho}}\right|^{\rho}
\end{aligned}
$$

as far as

$$
\left(\frac{v^{(m)}}{m !} \bigodot E_{k}\right)_{\alpha}^{\beta}=\frac{v^{\beta-\alpha}}{(\beta-\alpha) !}
$$

Due to the Hölder inequality

$$
\begin{aligned}
\left|\sum_{\beta \gg \alpha} \frac{A_{\beta}^{\alpha^{\prime}}}{(\beta-\alpha) ! 1 / \rho} \frac{v^{\beta-\alpha}}{(\beta-\alpha) !^{1 / \varrho}}\right|^{\rho} \leq \sum_{\beta \gg \alpha} \frac{\left|A_{\beta}^{\alpha^{\prime}}\right| \rho}{(\beta-\alpha) !}\left(\sum_{\beta \gg \alpha} \frac{\left|v^{\beta-\alpha}\right|^{\varrho}}{(\beta-\alpha) !}\right)^{\varrho} \\
\quad=\sum_{\beta \gg \alpha} \frac{\left|A_{\beta}^{\alpha^{\prime}}\right| \rho}{(\beta-\alpha) !}\left(\frac{\|v\|_{\varrho}^{m \varrho}}{m !}\right)^{\rho / \varrho} .
\end{aligned}
$$

Therefore

$$
\left\|A\left(\frac{v^{(m)}}{m !} \bigodot E_{k}\right)\right\|_{\rho}^{\rho} \leq \sum_{\beta, \alpha^{\prime}} \frac{\left|A_{\beta}^{\alpha^{\prime}}\right|^{\rho}}{\beta !\left(p^{\prime} !(m+k) !\right)^{\rho-1}}\left(\begin{array}{c}
m+k \\
k
\end{array}\right)^{\rho-1} \sum_{\beta \gg \alpha}\left(\begin{array}{c}
\beta \\
\alpha
\end{array}\right)\|v\|_{\varrho}^{m \rho}=
$$




$$
\sum_{\beta, \alpha^{\prime}} \frac{\left|A_{\beta}^{\alpha^{\prime}}\right|^{\rho}}{\beta !\left(p^{\prime} !(m+k) !\right)^{\rho-1}}\left(\begin{array}{c}
m+k \\
k
\end{array}\right)^{\rho}\|v\|_{\varrho}^{m \rho}=\|A\|_{\rho}^{\rho}\left(\begin{array}{c}
m+k \\
k
\end{array}\right)^{\rho}\|v\|_{\varrho}^{m \rho} .
$$

Corollary 1. For any nonnegative integer numbers $m, q^{\prime}$ and $A \in M_{n^{\prime}, n}\left(q^{\prime}\right.$, $m ; F), v^{i} \in M_{n, n}(1,0 ; F), i=1,2, \ldots, m$ the following inequality

$$
\left\|A \frac{v^{1} \odot v^{2} \odot \ldots \odot v^{m}}{m !}\right\|_{\rho} \leq\|A\|_{\rho}\left\|v^{1}\right\|_{\varrho}\left\|v^{2}\right\|_{\varrho} \ldots\left\|v^{m}\right\|_{\varrho}
$$

is valid.

\section{The Radius of Absolute Convergence}

We consider multivariate power series only with respect to the origin. Let us consider any multivariate power series $(1)$, where $a_{\alpha} \in F, z=\left(z_{1}, z_{2}, \ldots, z_{n}\right)$, $z_{1}, z_{2}, \ldots, z_{n^{-}}$are variables over $F$. We represent this power series in the form

$$
\sum_{\alpha \in I_{n}} a_{\alpha} z^{\alpha}=\sum_{m=0}^{\infty} A(m) \frac{z^{(m)}}{m !}
$$

, where $A(m) \in M(0, m ; F), A(m)_{\alpha}^{0}=a_{\alpha} \alpha$ !, $z$ is the corresponding column vector.

Definition 3. Power series (2) is said to be absolute convergent at $v \in F^{n}$ if the positive series $\sum_{\alpha \in I_{n}}\left|a_{\alpha} v^{\alpha}\right|$ converges.

Let $R_{\varrho}=\frac{1}{r_{\varrho}}$, where $r_{\varrho}=\varlimsup_{m \rightarrow \infty}\|A(m)\|_{\rho}^{\frac{1}{m}}$, and $R_{\varrho, c}=R_{\varrho} n^{\frac{\rho-1}{\rho}}=R_{\varrho} n^{\frac{1}{\varrho}}$.

Theorem 2. Power series (2) is absolute convergent at $v \in F^{n}$ whenever $\|v\|_{\varrho}<R_{\varrho}$ and for any $R>R_{\varrho, c}$ there exists such $\bar{v} \in F^{n}$ that $\|\bar{v}\|_{\varrho}=R$ and power series (2) is absolute divergent at $\bar{v}$.

Proof. It is easy to check that $\sum_{|\alpha|=m} \frac{\left|z^{\alpha}\right| \varrho}{\alpha !}=\frac{\|z\|_{e}^{m \varrho}}{m !}$. For any $m, A=A(m)$ due to the Hölder inequality one has

$$
\begin{aligned}
\sum_{|\alpha|=m}\left|A_{\alpha}^{0} \frac{z^{\alpha}}{\alpha !}\right| \leq\left(\sum_{|\alpha|=m} \frac{\left|A_{\alpha}^{0}\right|^{\rho}}{\alpha !}\right)^{\frac{1}{\rho}}\left(\sum_{|\alpha|=m} \frac{\left|z^{\alpha}\right|^{\varrho}}{\alpha !}\right)^{\frac{1}{\varrho}} \\
\quad=\left(\sum_{|\alpha|=m} \frac{\left|A_{\alpha}^{0}\right|^{\rho}}{\alpha !}\right)^{\frac{1}{\rho}} \frac{\|z\|_{\varrho}^{m}}{m !^{\frac{1}{\varrho}}}=\left(\sum_{|\alpha|=m} \frac{\left|A_{\alpha}^{0}\right|^{\rho}}{\alpha ! m !^{\rho-1}}\right)^{\frac{1}{\rho}}\|z\|_{\varrho}^{m}=\|A(m)\|_{\rho}\|z\|_{\varrho}^{m} .
\end{aligned}
$$


Therefore

$$
\sum_{m=0}^{\infty} \sum_{|\alpha|=m}\left|a_{\alpha} z^{\alpha}\right| \leq \sum_{m=0}^{\infty}\|A(m)\|_{\rho}\|z\|_{\varrho}^{m}
$$

The ordinary number series $\sum_{m=0}^{\infty}\|A(m)\|_{\rho}\|z\|_{\varrho}^{m}$ converges at $z=v$ whenever $\|v\|_{\varrho}<R_{\varrho}=1 / \varlimsup_{m \rightarrow \infty}\|A(m)\|_{\rho}^{\frac{1}{m}}$

It can be checked easily that the function $f(\rho)=\left(\sum_{|\alpha|=m} b_{\alpha}^{\rho} c_{\alpha}\right)^{\frac{1}{\rho}}$ is a decreasing function on $[1,+\infty)$, where $b_{\alpha}, c_{\alpha}$ are positive numbers, and therefore

$$
f(1)=\sum_{|\alpha|=m} b_{\alpha} c_{\alpha} \geq\left(\sum_{|\alpha|=m} b_{\alpha}^{\rho} c_{\alpha}\right)^{\frac{1}{\rho}}=f(\rho)
$$

Now let us assume that $R>R_{\varrho, c}$, that is $R n^{\frac{-1}{\varrho}} \overline{\lim }_{m \rightarrow \infty}\|A(m)\|_{\rho}^{\frac{1}{m}}>1$. It implies existence of such subsequence $\left\{m_{k}\right\}$ of natural numbers for which

$$
R n^{\frac{-1}{\varrho}}\left(\sum_{|\alpha|=m_{k}} \frac{\left|a_{\alpha} \alpha !\right|^{\rho}}{\alpha !\left(m_{k} !\right)^{\rho-1}}\right)^{\frac{1}{m_{k} \rho}}>1
$$

One can represent this inequality in the form

$$
\left(\sum_{|\alpha|=m_{k}}\left(\frac{\left|a_{\alpha}\left(R n^{\frac{-1}{\varrho}}\right)^{m_{k}}\right|}{\left(\begin{array}{c}
m_{k} \\
\alpha
\end{array}\right)}\right)^{\rho}\left(\begin{array}{c}
m_{k} \\
\alpha
\end{array}\right)\right)^{\frac{1}{m_{k} \rho}}>1
$$

that is

$$
\left(\sum_{|\alpha|=m_{k}}\left(\frac{\left|a_{\alpha}\left(R n^{\frac{-1}{\varrho}}\right)^{m_{k}}\right|}{\left(\begin{array}{c}
m_{k} \\
\alpha
\end{array}\right)}\right)^{\rho}\left(\begin{array}{c}
m_{k} \\
\alpha
\end{array}\right)\right)^{\frac{1}{\rho}}>1
$$

Therefore the series

$$
\sum_{k=1}^{\infty}\left(\sum_{|\alpha|=m_{k}}\left(\frac{\left|a_{\alpha}\left(R n^{\frac{-1}{\varrho}}\right)^{m_{k}}\right|}{\left(\begin{array}{c}
m_{k} \\
\alpha
\end{array}\right)}\right)^{\rho}\left(\begin{array}{c}
m_{k} \\
\alpha
\end{array}\right)\right)^{\frac{1}{\rho}}
$$

diverges and due to (3) the series

$$
\sum_{k=1}^{\infty} \sum_{|\alpha|=m_{k}}\left|a_{\alpha}\left(R n^{\frac{-1}{\varrho}}\right)^{m_{k}}\right|
$$


diverges as well. It implies divergence of $(2)$ at $\bar{v}=\left(R n^{\frac{-1}{\varrho}}, R n^{\frac{-1}{\varrho}}, \ldots, R n^{\frac{-1}{\varrho}}\right) \in$ $F^{n}$, for which $\|\bar{v}\|_{\varrho}=R$

Here is an estimation for $R_{\varrho}$.

Proposition 6. For the radius of absolute convergence $R_{\varrho}$ of power series (2) the following equalities

$$
R_{\varrho}=1 / \varlimsup_{m \rightarrow \infty} \max _{|\alpha|=m}\left|a_{\alpha}\left(\frac{\alpha !}{m !}\right)^{\frac{1}{\varrho}}\right|^{\frac{1}{m}}=1 / \varlimsup_{\lim _{m \rightarrow \infty}}\left(\frac{e}{m}\right)^{\frac{1}{\varrho}}\left(\max _{|\alpha|=m}\left|a_{\alpha} \alpha !^{\frac{1}{\varrho}}\right|\right)^{\frac{1}{m}}
$$

and inequalities

$$
R_{\infty} \leq R_{\varrho} \leq n^{\frac{1}{\varrho}} R_{\infty}
$$

are true.

Proof. Due to the definition

$$
\|A(m)\|_{\rho}=\left(\sum_{|\alpha|=m} \frac{\left|A(m)_{\alpha}^{0}\right|^{\rho}}{\alpha ! m ! \rho-1}\right)^{\frac{1}{\rho}}=\left(\sum_{|\alpha|=m}\left|a_{\alpha}\right|^{\rho}\left(\frac{\alpha !}{m !}\right)^{\rho-1}\right)^{\frac{1}{\rho}}
$$

Therefore it is clear that

$$
\max _{|\alpha|=m}\left|a_{\alpha}\left(\frac{\alpha !}{m !}\right)^{\frac{\rho-1}{\rho}}\right| \leq\|A(m)\|_{\rho} \leq \max _{|\alpha|=m}\left|a_{\alpha}\left(\frac{\alpha !}{m !}\right)^{\frac{\rho-1}{\rho}}\right|\left(\begin{array}{c}
m+n-1 \\
n-1
\end{array}\right)^{\frac{1}{\rho}}
$$

as far as $\sum_{|\alpha|=m} 1=\left(\begin{array}{c}m+n-1 \\ n-1\end{array}\right)$.

It implies that $r_{\varrho}=\varlimsup_{m \rightarrow \infty}\left(\max _{|\alpha|=m}\left|a_{\alpha}\left(\frac{\alpha !}{m !}\right)^{\frac{1}{\varrho}}\right|\right)^{\frac{1}{m}}$ that is

$$
R_{\varrho}=1 / \varlimsup_{m \rightarrow \infty}\left(\max _{|\alpha|=m}\left|a_{\alpha}\left(\frac{\alpha !}{m !}\right)^{\frac{1}{\varrho}}\right|\right)^{\frac{1}{m}}
$$

Due to the Stirling formula $m !=\sqrt{2 \pi m}\left(\frac{m}{e}\right)^{m} e^{\frac{\theta_{m}}{12 m}}$, where $0<\theta_{m}<1$, the above expression for $R_{\varrho}$ can be rewritten as

$$
R_{\varrho}=1 / \overline{\lim }_{m \rightarrow \infty}\left(\frac{e}{m}\right)^{\frac{1}{\varrho}}\left(\max _{|\alpha|=m}\left|a_{\alpha} \alpha !^{\frac{1}{\varrho}}\right|\right)^{\frac{1}{m}}
$$

as well. It is the proof of the stated equalities.

As to the inequalities one can notice that due to the Stirling formula

$$
\lim _{m \rightarrow \infty}\left(\frac{\left[\frac{m}{n}\right] !^{n}}{m !}\right)^{\frac{1}{m}}=\frac{1}{n}
$$


, where $\left[\frac{m}{n}\right]$ stands for the integer part of $\frac{m}{n}$, and

$$
\max _{|\alpha|=m}\left|a_{\alpha}\right|\left(\frac{\left[\frac{m}{n}\right] !^{n}}{m !}\right)^{\frac{\rho-1}{\rho}} \leq \max _{|\alpha|=m}\left|a_{\alpha}\left(\frac{\alpha !}{m !}\right)^{\frac{\rho-1}{\rho}}\right| \leq \max _{|\alpha|=m}\left|a_{\alpha}\right|
$$

which implies

$$
r_{\infty} n^{\frac{-1}{\varrho}} \leq r_{\varrho} \leq r_{\infty}
$$

that is

$$
R_{\infty} \leq R_{\varrho} \leq n^{\frac{1}{\varrho}} R_{\infty}
$$

Corollary 2. The equality

$$
R_{\varrho}=\lim _{m \rightarrow \infty} \frac{\max _{|\alpha|=m}\left|a_{\alpha}\left(\frac{\alpha !}{m !}\right)^{\frac{1}{\varrho}}\right|}{\max _{|\alpha|=m+1}\left|a_{\alpha}\left(\frac{\alpha !}{(m+1) !}\right)^{\frac{1}{\varrho}}\right|}
$$

is true provided that the limit exists.

Now let us consider some examples of multivariate power series to demonstrate effectiveness the introduced radius of absolute convergence. All considered examples will be multivariate power series in two real variables $x=\left(x_{1}, x_{2}\right)$.

Example 2. Let us consider the power series

$$
\sum_{m=0}^{\infty}\left(x_{1} x_{2}\right)^{m}=\sum_{m=0}^{\infty} A(2 m) \frac{x^{(2 m)}}{(2 m) !},
$$

all components of $A(2 m)$ are zero except for $A(2 m)_{(m, m)}^{0}=m !^{2}$. This power series converges at $x=\left(x_{1}, x_{2}\right)$ if and only if $\left|x_{1} x_{2}\right|<1$. For the norm one has

$$
\|A(2 m)\|_{\rho}=\left(\frac{(m !)^{2 \rho}}{m ! m !(2 m) ! \rho-1}\right)^{\frac{1}{\rho}}=\left(\frac{m !^{2}}{(2 m) !}\right)^{\frac{\rho-1}{\rho}}
$$

It implies that

$$
r_{\varrho}=\varlimsup_{m \rightarrow \infty}\|A(2 m)\|_{\rho}^{\frac{1}{2 m}}=\varlimsup_{m \rightarrow \infty}\left(\frac{m !^{2}}{(2 m) !}\right)^{\frac{\rho-1}{2 m \rho}}=2^{\frac{-1}{\varrho}}
$$

due to the Stirling formula.

So $R_{\varrho}=2^{\frac{1}{\varrho}}$ and $R_{\varrho, c}=2^{\frac{2}{\varrho}}$. In the layer of indeterminacy there are points where the series is absolute convergent and there are points where the series is 
absolute divergent. The obtained $R_{\varrho}=2^{\frac{1}{\varrho}}$ is the best one, it can't be increased.

Example 3. Let $\sum_{i, j=0}^{\infty} x_{1}^{i} x_{2}^{j}=\sum_{m=0}^{\infty} A(m) \frac{x^{(m)}}{m !}$, where $A(m)_{(i, m-i)}^{0}=$ $i !(m-i)$ ! It absolute converges at $x=\left(x_{1}, x_{2}\right)$ if and only if $\left|x_{1}\right|<1$ and $\left|x_{2}\right|<1$.

$$
\|A(m)\|_{\rho}=\left(\sum_{i=0}^{m} \frac{\left|A(m)_{(i, m-i)}^{0}\right|^{\rho}}{(i) !(m-i) !(m) ! \rho-1}\right)^{\frac{1}{\rho}}=\left(\sum_{i=0}^{m}\left(\begin{array}{c}
m \\
i
\end{array}\right)^{1-\rho}\right)^{\frac{1}{\rho}}
$$

Due to the inequalities $1 \leq \sum_{i=0}^{m}\left(\begin{array}{c}m \\ i\end{array}\right)^{1-\rho} \leq m+1$ one has

$$
r_{\varrho}=\varlimsup_{m \rightarrow \infty}\|A(m)\|_{\rho}^{\frac{1}{m}}=1,
$$

that is $R_{\varrho}=1$ and $R_{\varrho, c}=2^{\frac{1}{\varrho}}$. In this case the obtained $R_{\varrho}$ is the best one, it can't be increased. In the case of $\varrho=\infty$ the power series is absolute divergent at all points of the layer $1=R_{\infty} \leq\|x\|_{\infty} \leq R_{\infty, c}=2$

We have no example of a multivariate power series for which the corresponding $R_{\varrho}$ is not the best one.

The introduced radius of absolute convergence may be useful in the case of multivariate Loran power series

$$
\sum_{\alpha \in Z^{n}} a_{\alpha} z^{\alpha}
$$

as well, where $Z$ stands for the set of integer numbers, $a_{\alpha} \in F$.

For that one can represent multivariate Loran power series (4) in the following (unique) form.

$$
\sum_{\alpha \in Z^{n}} a_{\alpha} z^{\alpha}=\sum_{\sigma} \sum_{m=0}^{\infty} A(\sigma, m) \frac{z_{\sigma}^{(m)}}{m !}
$$

, where $\sigma$ runs the set of all functions from $\{1,2, \ldots, n\}$ to $\{-1,1\}, z_{\sigma}=$ $\left(z_{1}^{\sigma(1)}, z_{2}^{\sigma(2)}, \ldots, z_{n}^{\sigma(n)}\right), A(\sigma, m) \in \operatorname{Mat}(0, m ; F)$ and $A(\sigma, m)_{\alpha}^{0}=0$ whenever $\sigma^{-1}(-1)$ is not a subset of $\operatorname{supp}(\alpha)=\left\{i: \alpha_{i} \neq 0\right\}$. Each multivariate power series $\sum_{m=0}^{\infty} A(\sigma, m) \frac{z_{\sigma}^{(m)}}{m !}$ can be investigated for absolute convergence separately and then decided if they have a common point of absolute convergence.

For example, if a power series is given as

$$
\sum_{\alpha \in I_{n}} a_{\alpha}^{+} z^{\alpha}+\sum_{\alpha \in I_{n} \backslash 0} a_{\alpha}^{-} z^{-\alpha}
$$


and $R_{\varrho}^{+}\left(R_{\varrho}^{-}\right)$is radius of absolute convergence for $\sum_{\alpha \in I_{n}} a_{\alpha}^{+} z^{\alpha}$ (respect.

$$
\left.\sum_{\alpha \in I_{n} \backslash\{0\}} a_{\alpha}^{-} z^{-\alpha}\right)
$$

then the Loran series is absolute convergent on a nonempty open set whenever $R_{\varrho}^{+} R_{\varrho}^{-}>n^{\frac{2(\rho-1)}{\rho}}=n^{\frac{2}{\varrho}}$.

At the end we would like to state the following problems.

Problem 1. An example of a multivariate power series for which the corresponding $R_{\varrho}$ is not the best one, that is it can be increased.

Problem 2. Consider any multivariate power series (2), for which

$$
\sum_{m=0}^{\infty} H_{m}(z)
$$

where $H_{m}(z)=\sum_{|\alpha|=m} a_{\alpha} z^{\alpha}$, is absolute convergent in some neighborhood of zero. Does it imply that the original series $\sum_{\alpha} a_{\alpha} z^{\alpha}$ is absolute convergent in some neighborhood of zero as well?

Problem 3. Consider any multivariate power series (2), for each nonnegative $m$ multi-linear map

$$
\left(F^{n}\right)^{m} \longrightarrow F:\left(v^{1}, v^{2}, \ldots, v^{m}\right) \longmapsto A(m) \frac{\left(v^{1} \odot v^{2} \odot \ldots \bigodot v^{m}\right)}{m !}
$$

and its norm $\|A(m)\|([5])$ defined by

$$
\|A(m)\|=\sup _{\left\|v^{1}\right\|_{\varrho}=\left\|v^{2}\right\|_{\varrho}=\ldots=\left\|v^{m}\right\|_{\varrho}=1}\left|A(m) \frac{\left(v^{1} \bigodot v^{2} \bigodot \ldots \bigodot v^{m}\right)}{m !}\right|
$$

According to Corollary 1 for each $m$ the inequality $\|A(m)\| \leq\|A(m)\|_{\rho}$ is true. Is it true that

$$
\varlimsup_{m \rightarrow \infty}\|A(m)\|^{\frac{1}{m}}=\varlimsup_{m \rightarrow \infty}\|A(m)\|_{\rho}^{\frac{1}{m}} \text { ? }
$$

\section{References}

[1] B.V. Shabat, Introduction to Complex Analysis, Part II, Multivariate Functions, Nauka, Moscow (1976), In Russian.

[2] Ural Bekbaev, A matrix representation of composition of polynomial maps, arXiv: math 0901.3179v3. 
[3] Ural Bekbaev, Matrix Representations for symmetric and antisymmetric multi-linear maps, arXiv:1010.2579, math.RA.

[4] Ural Bekbaev, A radius of absolute convergence for power series in many variables, arXiv:1001.0622 math. CV (math.AC).

[5] H. Cartan, Calcul Différntiel, Forms Différentielles, Hermann, Paris (1967). 